\title{
Detection and significance of glial fibrillary acidic protein antibody in autoimmune astocytopathy and related diseases
}

\author{
Jiehong Huang ${ }^{1}$, Wenyao Huang ${ }^{1}$, Ruisi Zhou ${ }^{1}$, Wenhui Lin $^{1}$, Ting Chen ${ }^{1}$, Youming Long ${ }^{1,2,3}$ \\ ${ }^{1}$ Second Clinical College, Guangzhou Medical University, Guangzhou, China; ${ }^{2}$ Department of Neurology, The Second Affiliated Hospital of \\ Guangzhou Medical University, Guangzhou, China; ${ }^{3}$ Institute of Neuroscience and The Second Affiliated Hospital of Guangzhou Medical \\ University, Key Laboratory of Neurogenetics and Channelopathies of Guangdong Province and the Ministry of Education of China, Collaborative \\ Innovation Center for Neurogenetics and Channelopathies, Guangzhou, China \\ Contributions: (I) Conception and design: Y Long; (II) Administrative support: None; (III) Provision of study materials or patients: None; (IV) \\ Collection and assembly of data: None; (V) Data analysis and interpretation: None; (VI) Manuscript writing: All authors; (VII) Final approval of \\ manuscript: All authors. \\ Correspondence to: Youming Long. Department of Neurology, Institute of Neuroscience, The Second Affiliated Hospital of Guangzhou Medical \\ University, Key Laboratory of Neurogenetics and Channelopathies of Guangdong Province and the Ministry of Education of China, Collaborative \\ Innovation Center for Neurogenetics and Channelopathies, 250\# Changgang East Road, Guangzhou 510260, China. Email: youminglong@126.com.
}

\begin{abstract}
Autoimmune glial fibrillary acidic protein astrocytopathy (GFAP-A) is an antibody-related astrocytic disease for which a specific GFAP antibody serves as a biological marker. Indeed, cerebral spinal fluid positive and/or seropositivity for GFAP is an important basis for its diagnosis. However, because patients with autoimmune encephalitis or demyelinating diseases can have a similar antibody profile, termed overlapping autoimmune syndrome, it remains a challenge for clinicians to diagnose and suitably classify autoimmune GFAP-A. To further understand the significance of GFAP antibody detection in neuroimmune diseases, this article discusses GFAP antibodies in autoimmune GFAP-A, progress for detection of GFAP antibodies, diagnostic significance of GFAP antibodies in prototypical disease, as well as overlapping syndrome.
\end{abstract}

Keywords: Astrocyte; antibody; aquaporin 4; glial fibrillary acidic protein (GFAP); overlapping syndrome

Submitted Jan 27, 2019. Accepted for publication May 10, 2019. Published online Aug 05, 2019.

doi: $10.21037 /$ atm-19-330

View this article at: http://dx.doi.org/10.21037/atm-19-330

\section{Glial fibrillary acidic protein (GFAP) antibody in autoimmune GFAP astrocytopathy (GFAP-A)}

In 2016, Fang et al. first proposed a new independent disease entity, human autoimmune GFAP-A. They used tissue-based assay (TBA) and cell-based assays (CBA) to detect GFAP antibodies in cerebrospinal fluid (CSF) and serum, and identified that specific GFAP antibodies are biomarkers of autoimmune GFAP-A $(1,2)$. The phenotypes encountered among those with serum positivity were heterogenous. Serum GFAP antibody positivity can occur for a variety of conditions, such as traumatic brain injury, brain tumors, autism, lead exposure, or diabetes (3-6). Moreover, in Fang's study (2), they found that GFAPIgG among $0.5 \%$ to $1.5 \%$ of controls serums, although never by both tissue and cell-based testing, and just once in control CSF. The specificity of GFAP antibody in serum was significantly worse than that of GFAP antibody in CSF. In a study at the Mayo Clinic, 49 of the 102 patients with GFAP-A were tested for serum and CSF, and 45 patients had positive GFAP-IgG in CSF, but only 22 were seropositive. This difference is statistically significant. This indicates that GFAP antibody positive in CSF in patients with GFAP-A has high specificity and sensitivity compared with serum (2,7-9). Therefore, at present, GFAP antibody positivity in CSF is more clinically significant than seropositivity for diagnosis of GFAP-A $(1,2,10)$. Therefore, when we found that GFAP antibody was positive in the serum of patients, we should carry out lumbar puncture at the same time, obtained CSF and detected GFAP antibody 


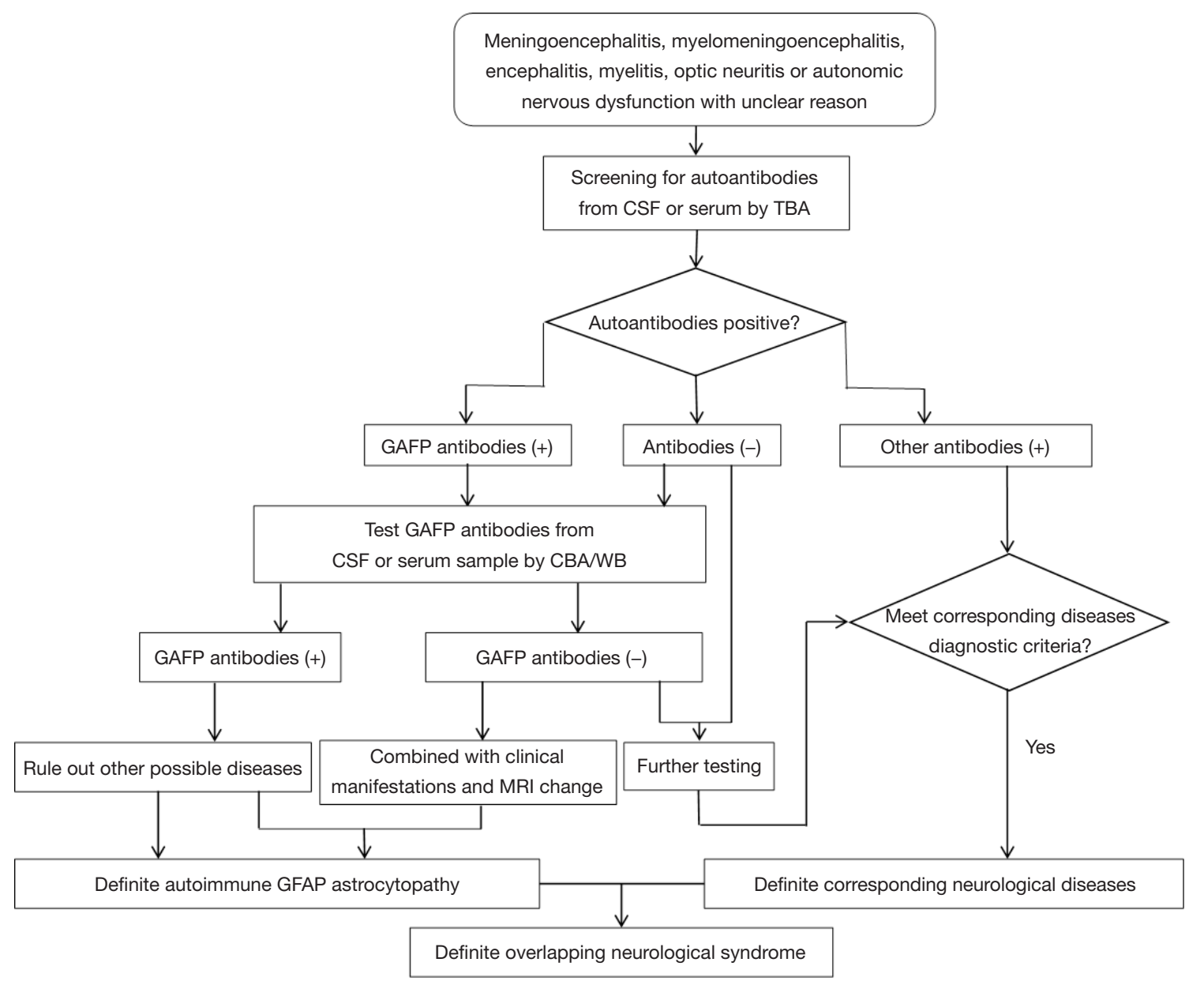

Figure 1 Diagnostic flow chart of GFAP astrocytopathy. CSF, cerebrospinal fluid; TBA, tissue-based assay; GFAP, glial fibrillary acidic protein; CBA, cell-based assay; WB, western blot; MRI, magnetic resonance imaging.

in CSF in order to improve the diagnostic rate. Although the specificity of GFAP antibodies in serum is not as good as that of CSF, the positive result of GFAP antibody in serum samples is still a meaningful result. In the diagnosis of GFAP-A, we recommend GFAP antibody positive in CSF as the main diagnostic criteria. When patients only have serum GFAP antibodies, we should evaluate the patient's clinical manifestations and magnetic resonance imaging (MRI) characteristics. At the same time, we should exclude other possible diseases, such as traumatic brain injury, brain tumors, autism, lead exposure, or autoimmune diabetes (see Figure 1 diagnostic flow chart of GFAP-A).

GFAP was an intermediate filament protein found in the cytoplasm of astrocytes, and its mRNA has seven different alternative splicing products. The most abundant GFAP mRNA isoform is GFAP $\alpha$, which was the first to be identified. Indeed, most articles describing GFAP expression patterns and regulation are primarily concerned with GFAP- $\alpha$ (11). GFAP subtypes $\alpha, \delta / \varepsilon$, and $\kappa$ contain the same head and coil domain, but the $\mathrm{C}$-terminal tail is divergent and may have differential functions (11). GFAP- $\varepsilon$ is expressed during early development, most abundantly in neural progenitor cells in the periventricular zone, hippocampus, and central spinal cord (12). GFAP- $\kappa$ expression is most abundant during early fetal development, and then rapidly decreases as GFAP- $\alpha$ and GFAP- $\varepsilon$ subtypes proportionally increase (13). There are more than seven isoforms of GFAP antibodies, The GFAP antibody isoforms in serum and/or CSF of patients with GFAP astrocytosis have $\alpha, \gamma, \delta / \varepsilon$ and $\kappa$. In a study by Mayo team et al., $100 \%(102 / 102)$ of the 102 patients diagnosed with GFAP-A were positive for GFAP $\alpha$-IgG in serum and/or 
CSF, $81 \%$ (76/94). Patients with GFAP- $\varepsilon-\operatorname{IgG}$ positive patients, $54 \%$ of patients with GFAP-к-IgG and GFAP- $\varepsilon$ IgG double positive. GFAP antibody subtypes were mainly GFAP $-\alpha-\operatorname{IgG}$, followed by GFAP- $\varepsilon$ and GFAP- $\kappa-\operatorname{IgG}$, and our group found that 21 patients were positive for GFAP- $\alpha$ IgG and/or GFAP $\varepsilon-\operatorname{IgG}$. Among them, 16 patients (76.2\%) were double positive for GFAP $\alpha-\operatorname{IgG}$ and GFAP- $\varepsilon-\operatorname{IgG}$, and 5 patients $(23.8 \%)$ were only positive for GFAP $\varepsilon$ IgG. The results of the Italian research team showed that the antibody subtypes were mainly GFAP- $\alpha-\operatorname{IgG}(100 \%)$, followed by GFAP- $\alpha$ - $\operatorname{IgG}$ and $\delta$-IgG (14/22, 64\%).

The GFAP antibody associated with astrocytopathy displays a similar pattern of immunofluorescence (IF) as aquaporin 4 (AQP4)-IgG, binding to pia and subpia, but differences between them also exist as follows: GFAP antibodies were mainly distributed in the cell body and terminal processes of each layer (molecular layer, white matter and granular layer), and distributed radially in the cerebellar molecular layer, whereas AQP4-IgG mainly binds to locations around the microvessels and the Virchow-Robin space with only around microvessels in cerebellum and Virchow-Robin space distribution at the junction of two molecular layers. Additionally, differences in immunostaining are that GFAP-IgG was confined to pial, subpial and subventricular regions, while AQP4-IgG can be detected in the center of semiovale, basal ganglia, paraventricular, thalamus and brainstem margin, and cerebellum can be involved in a few patients $(7,8,14)$.

The pathogenesis of autoimmune GFAP-A has not been elucidated. Notably, GFAP within intact astrocytes is difficult to contact with GFAP antibodies, because GFAP is an intracellular protein. Fang et al. speculated that autoimmune GFAP-A is associated with GFAP peptidespecific cytotoxic T cells (1). Iorio et al. suggested that GFAP antibodies may be a surrogate marker for cytotoxic $T$ cell-mediated autoimmune responses (15). For the time being, positivity for GFAP antibody in CSF and/or serum, as primarily detected by IF, CBA, and western blot (7), is an important indicator for the diagnosis of autoimmune GFAP-A.

\section{Progress in detection of GFAP antibodies}

Identification of specific diagnostic and pathogenic biomarkers has been a primary focus of clinical research on central nervous system (CNS) autoimmune diseases. Early detection of specific antibodies facilitates the clinician's timely diagnosis and treatment, which can avoid irreversible disability and even paralysis. In contrast to AQP4, GFAP is an intracellular protein, which limits available detection methods.

Primary detection methods for GFAP antibodies include TBA, CBA, and western blot. TBA has high sensitivity and certain detection value for unknown antibodies, but its specificity is less than CBA or western blot. CBA has higher specificity for identifying subtypes, but inevitably has a high false-negative rate because GFAP is an intracellular protein that is not easily accessed by antibodies $(1,8,15)$. Moreover, because more than seven different subtypes of GFAP antibodies exist, the application of CBA for all of these isomers remains difficult.

Notably, GFAP- $\alpha$ IgG was found in $100 \%$ of the 102 patients in the Mayo Clinic study, and 22 patients from European tertiary referral hospitals exhibited $100 \%$ frequency GFAP- $\alpha$ positivity, with $63.6 \%$ exhibiting both GFAP- $\alpha$ and GFAP- $\delta$, whereas none exhibited the GFAP- $\delta$ isoform alone $(2,15)$. The GFAP- $\alpha$ subtype is the most abundant and widely distributed, and it has been found that the sensitivity of detecting GFAP- $\alpha$ antibody is higher than that of other subtypes in the reported laboratory results. However, amongst 19 Chinese patients who underwent CSF testing, we identified 14 who were positive for GFAP $-\alpha \operatorname{IgG}$ and five who were only positive for GFAP- $\varepsilon$ $\operatorname{IgG}$, indicating novel results that should be confirmed by other laboratories in the future (8). Because studies with large sample sizes are lacking, current in-depth discussion of this issue has not yet been reported. Western blot, which has high specificity for GFAP antibodies, is usually used to confirm experimental results (16). At present, the Mayo Clinic (USA), Second Affiliated Hospital of Guangzhou Medical University (China), and Catholic University (Italy) have reported GFAP-A. Detection methods used by these three research centers are similar (all used TBA, CBA, and western blot), but there are also some differences such as sample size and laboratory technology level between different research centers. Thus, detection rates for GFAP antibodies were different. However, the sensitivity and specificity of GFAP antibody detection in CSF were usually higher than in serum tests.

Because each method has its own advantages and disadvantages, how do we solve this contradiction? Similar to the detection of intracellular protein antibodies for $\mathrm{HU}$ and $\mathrm{Ri}$, we suggest first screening by TBA, and then using western blot to detected GFAP antibodies in patient serum and/or CSF. On the one hand, TBA is sensitive and the use of western blot makes up for shortcoming of TBA in 
specific detection; on the other, TBA has great value for screening unknown antibodies. Although GFAP antibodies may have difficulties in binding to intracellular antigens, resulting in a decrease in CBA detection, we recommend CBA as the preferred method for GFAP antibody detection. The causes of the GFAP antibody identification using CBA: firstly, CBA is more economical than the western blot, and is convenient to be popularized in the clinic; secondly, due to the strong specificity of the CBA, the CBA can identify the sample determined by TBA.

Detection of antibodies is important for the diagnosis of autoimmune encephalopathy, especially antibodydependent CNS autoimmune diseases. However, because GFAP antibodies often overlap with other autoantibodies, suitable diagnostic and classification criteria are needed for the adoption of appropriate treatment. Thus far, we have suggested that detection of GFAP antibody in both serum and CSF of patients with unknown meningoencephalitis, encephalitis, myelitis, optic neuritis, or autonomic nervous dysfunction is needed (7). According to current data, GFAP antibody positivity in CSF exhibits the highest clinical sensitivity and specificity for diagnosis of GFAP-A, but we don't know yet if the increased antibodies and proteins in CSF were synthesized intrathecally or not, therefore, we recommend paired examinations of CSF and serum, analysis of patients' blood-CSF barrier function and intrathecal synthesis. Differentiating the source of specific antibodies in CSF can helps us to analyze the pathogenesis of autoimmune encephalitis.

\section{Diagnosis of overlapping syndrome by detection of GFAP antibodies}

Overlapping syndrome is a clinical syndrome that occurs in antibody-mediated inflammatory diseases (17). Two or more autoimmune antibodies are often present in the serum or CSF of patients with autoimmune overlapping syndrome. In a Mayo Clinic study, Lennon et al. detected one or more coexisting antibodies in the serum or CSF of 41 out of 102 patients $(40 \%)$. The most common coexisting antibody was $\mathrm{N}$-methyl-D-aspartate receptorIgG (NMDAR-IgG) (22\%), followed by AQP4-IgG (10\%) $(1,2)$. Similar results were observed in a European tertiary referral hospital, whereby 5 out of 22 patients $(22.7 \%$ ) had coexisting antibodies (15). In our previous study, we also observed 10 patients with GFAP antibodies coexisting with other autoantibodies. However, our data indicated AQP4$\operatorname{IgG}(50 \%, 5$ of 10$)$ to be the most common overlapping antibody, which is different from the results of the Mayo study (17). However, although two or more immune mechanisms may occur in GFAP-A with overlapping syndrome, no further differences could be identified between the patients with and without overlapping syndrome, except for the age at onset, that is younger in patients with overlapping syndrome. Although identification of overlapping antibodies is common for GFAP-A, a lack of large-scale investigations means that differences in overlapping antibodies amongst different ethnicities are still unknown. Further research is therefore needed to analyse whether the overlapping antibodies mean overlapping with other CNS diseases or just are non-specific antibodies caused by other diseases, such as infection and tumor.

In our previous study, we found that two cases $(20 \%$, 2/10) of GFAP-A occurred separately from the time of occurrence of anti-NMDAR encephalitis or AQP4 astrocytopathy, which made it easy to identify and confirm the diagnosis. However, eight patients $(80 \%$, 8/10) exhibited GFAP antibodies with clinical and MRI features of autoimmune encephalitis or demyelinating disease simultaneously. According to the patients' clinical manifestations and positive results for an AQP4 antibody, five met the diagnostic criteria of NMOSD and were diagnosed as NMOSD, while the other three patients were diagnosed as autoimmune neuron antibody-positive encephalitis according to the corresponding diagnostic criteria (17). Notably, the phenotype of overlapping syndrome is different compared with the phenotype of prototypical disease, indicating that there may be more than two immunological pathogenic mechanisms in patients with overlapping syndrome. Although some researchers have reported individual cases of GFAP-A infection or prodromal infection, the relationship between infection and GFAP antibody has not been clearly documented. In the absence of unified diagnostic criteria or an international consensus for GFAP-A, identifying and distinguishing phenotypes with multiple overlapping autoantibodies has become an urgent problem.

So, how should we diagnose and rule out patients with suspected encephalitis? On the basis of current international consensus and diagnostic criteria for the diagnosis of NMOSD, NMOSD can be diagnosed from the patient's clinical manifestations and positivity for AQP4 antibody. Similarly, on the basis of the results of serum or CSF autoantibodies and GFAP antibody positivity, combined with the patient's current unexplained fever or tremor as well as other symptoms 
such as headache, encephalopathy, involuntary movement, long segment myelitis, optic nerve abnormalities, ataxia, mental and emotional abnormalities, epilepsy, autonomic nervous dysfunction and other meningeal-brain symptoms and signs, MRI features, typical enhancement of white matter vessels perpendicular to the ventricle, can be used for diagnosis of GFAP-A. Basic diagnostic procedures include: (I) detection of autoimmune antibodies, (II) combination with clinical manifestations described above, (III) MRI: involved areas include white matter, basal ganglia, brainstem, cerebellum, meninges, ventricles and even skulls and other exclusive examinations such as the screening for tumor factors and infection related factors. For the diagnosis of overlapping syndrome, we first examined (I) detection of autoimmune antibodies; (II) the presence of multiple autoantibodies, according to their corresponding diagnostic criteria for diagnosis; (III) GFAP antibody positivity combined with characteristic clinical manifestations of GFAP-A and MRI characteristics, to establish a diagnosis of GFAP-A; and then (IV) integrated the above diagnosis to establish a diagnosis of overlapping syndrome after excluding the precursor infection.

\section{Acknowledgments}

We thank Liwen Bianji, Edanz Group China (www. liwenbianji.cn/ac), for editing the English text of a draft of this manuscript.

Funding: This study was supported by the National Natural Science Foundation of China (No. 81771302), Science and Technology Program of Guangzhou, China (No. 201803010123), and the Science and Technology Plan Project of Guangdong Province (No. 2015A030302093).

\section{Footnote}

Provenance and Peer Review: This article was commissioned by the Guest Editors (Hai-Feng Li and Xiangjun Chen) for the series "Laboratory Investigations in Neuroimmunological Diseases and Their Clinical Significance" published in Annals of Translational Medicine. The article has undergone external peer review.

Conflicts of Interest: All authors have completed the ICMJE uniform disclosure form (available at https://atm.amegroups. com/article/view/10.21037/atm-19-330/coif). The series "Laboratory Investigations in Neuroimmunological Diseases and Their Clinical Significance" was commissioned by the editorial office without any funding or sponsorship. The authors have no other conflicts of interest to declare.

Ethical Statement: The authors are accountable for all aspects of the work in ensuring that questions related to the accuracy or integrity of any part of the work are appropriately investigated and resolved.

Open Access Statement: This is an Open Access article distributed in accordance with the Creative Commons Attribution-NonCommercial-NoDerivs 4.0 International License (CC BY-NC-ND 4.0), which permits the noncommercial replication and distribution of the article with the strict proviso that no changes or edits are made and the original work is properly cited (including links to both the formal publication through the relevant DOI and the license). See: https://creativecommons.org/licenses/by-nc-nd/4.0/.

\section{References}

1. Fang B, McKeon A, Hinson SR, et al. Autoimmune Glial Fibrillary Acidic Protein Astrocytopathy: A Novel Meningoencephalomyelitis. JAMA Neurol 2016;73:1297-307.

2. Flanagan EP, Hinson SR, Lennon VA, et al. Glial fibrillary acidic protein immunoglobulin $\mathrm{G}$ as biomarker of autoimmune astrocytopathy: Analysis of 102 patients. Ann Neurol 2017;81:298-309.

3. Kirkman NJ, Libbey JE, Sweeten TL, et al. How relevant are GFAP autoantibodies in autism and Tourette Syndrome? J Autism Dev Disord 2008;38:333-41.

4. Gomez-Tourino I, Camina-Darriba F, Otero-Romero I, et al. Autoantibodies to glial fibrillary acid protein and S100beta in diabetic patients. Diabet Med 2010;27:246-8.

5. Wei $P$, Zhang $W$, Yang LS, et al. Serum GFAP autoantibody as an ELISA-detectable glioma marker. Tumour Biol 2013;34:2283-92.

6. Zhang Z, Zoltewicz JS, Mondello S, et al. Human traumatic brain injury induces autoantibody response against glial fibrillary acidic protein and its breakdown products. PLoS One 2014;9:e92698.

7. Shan F, Long Y, Qiu W. Autoimmune Glial Fibrillary Acidic Protein Astrocytopathy: A Review of the Literature. Front Immunol 2018;9:2802.

8. Long Y, Liang J, Xu H, et al. Autoimmune glial fibrillary acidic protein astrocytopathy in Chinese patients: a retrospective study. Eur J Neurol 2018;25:477-83.

9. Chen NC, Lai PH, Fang HC, et al. Microscopic 
polyangiitis with an initial presentation of pontine infarction. Am J Med Sci 2012;344:163-5.

10. Dubey D, Hinson SR, Jolliffe EA, et al. Autoimmune GFAP astrocytopathy: Prospective evaluation of 90 patients in 1 year. J Neuroimmunol 2018;321:157-63.

11. Middeldorp J, Hol EM. GFAP in health and disease. Prog Neurobiol 2011;93:421-43.

12. Roelofs RF, Fischer DF, Houtman SH, et al. Adult human subventricular, subgranular, and subpial zones contain astrocytes with a specialized intermediate filament cytoskeleton. Glia 2005;52:289-300.

13. Blechingberg J, Lykke-Andersen S, Jensen TH, et al. Regulatory mechanisms for 3 '-end alternative splicing and polyadenylation of the Glial Fibrillary Acidic Protein, GFAP, transcript. Nucleic Acids Res 2007;35:7636-50.

14. Jarius S, Wildemann B. Aquaporin-4 antibodies

Cite this article as: Huang J, Huang W, Zhou R, Lin W, Chen T, Long Y. Detection and significance of glial fibrillary acidic protein antibody in autoimmune astocytopathy and related diseases. Ann Transl Med 2023;11(7):288. doi: 10.21037/ atm-19-330
(NMO-IgG) as a serological marker of neuromyelitis optica: a critical review of the literature. Brain Pathol 2013;23:661-83.

15. Iorio R, Damato V, Evoli A, et al. Clinical and immunological characteristics of the spectrum of GFAP autoimmunity: a case series of 22 patients. J Neurol Neurosurg Psychiatry 2018;89:138-46.

16. Liu T, Chen B, Yang H, et al. Screening for autoantibodies in inflammatory neurological syndrome using fluorescence pattern in a tissue-based assay: Cerebrospinal fluid findings from 793 patients. Mult Scler Relat Disord 2018;28:177-83.

17. Yang $\mathrm{X}, \mathrm{Xu} \mathrm{H}$, Ding M, et al. Overlapping Autoimmune Syndromes in Patients With Glial Fibrillary Acidic Protein Antibodies. Front Neurol 2018;9:251. 